\title{
İstanbul İli Avrupa Yakası Bazı Bölgelerinde Hava Kalitesinin Mikrobiyolojik Yoğunluk Açısından İncelenmesi
}

\author{
Sümeyye Aydın ${ }^{1}$, Hürrem Bayhan ${ }^{2 *}$, Gökhan Önder Ergüven ${ }^{3}$, Bahar İkizoglu ${ }^{4}$ \\ ${ }^{1}$ Koza Academy, Occupational Health and Safety Engineering Services Trade Limited Company, Istanbul, Turkey (ORCID: 0000-0003-3925-4027) \\ ${ }^{2}$ Yildiz Technical University, Faculty of Civil Engineering, Environmental Engineering Department, Istanbul, Turkey (ORCID: 0000-0003-4782-4683) \\ ${ }^{3}$ Munzur University, Tunceli Vocational School, Department of Chemistry and Chemical Process Technologies, Laboratory Technology Pr, Tunceli, Turkey \\ (ORCID: 0000-0003-1573-080X) \\ ${ }^{4}$ Suleyman Demirel Üniversity, Faculty of Engineering, Department of Environmental Engineering, Isparta, Turkey (ORCID: 0000-0002-6654-7303)
}

(İlk Geliş Tarihi 10 Şubat 2020 ve Kabul Tarihi 23 Mayıs 2020)

(DOI: 10.31590/ejosat.701133)

ATIF/REFERENCE: Aydın. S, Bayhan, H. \& Ergüven, G. Ö. (2020). İstanbul İli Avrupa Yakası Bazı Bölgelerinde Hava Kalitesinin Mikrobiyolojik Yoğunluk Açısından İncelenmesi. Avrupa Bilim ve Teknoloji Dergisi, (19), 334-343.

$\ddot{O} \mathbf{z}$

İstanbul'un dış havasının mikrobiyal faunası avrupa yakasına ait dört faklı bölgede (Bakırköy, Fatih, Başakşehir ve Esenler) aktif ve pasif sedimentasyon örnekleme metoduyla incelenmiştir. Meteorolojik faktörlere ilave olarak, kentleşme ve trafik yoğunluğu mikrobiyal yükleme için tespit edilmiştir. Dış hava örnekleme Nisan 2014-Kasım 2015 arasında yürütülmüştür. Sedimentasyon yöntemi standart plate count agar (PCA) kullanılarak hazırlanan petri kutularının kapakları açılarak yapılmışıı. PCA ortamı toplam bakterilerin belirlenmesinde kullanılırken mantarlar için malt ekstrakt agar (MEA) kullanılmıştır. Bu periyodun sonunda oluşan koloniler sayılmış ve hacim başına düşen toplam bakteri ve mantar sayısı belirlenmiştir. Hava sıcaklığı, bağıl nem, rüzgar hızı ve yönü de aynı zamanda kayıt edilmiş̧tir. Aktif örnekleme metodu sonuçlarına göre, en fazla bakteri sayıs $35^{\circ} \mathrm{C}$ inkübasyon sıcaklığında Başaksehir istasyonunda $222 \mathrm{KOB}$ (Koloni oluşturan birey) $/ \mathrm{m}^{3}$, en fazla mantar sayıs $20^{\circ} \mathrm{C}$ inkübasyon sıcaklığında da $286 \mathrm{KOB} / \mathrm{m}^{3}$ olarak belirlenmiştir. Belirlenen mikroorganizmalar için herhangi bir tür teşhis yapılmamıştır. Pasif örnekleme metodunda en fazla bakteri sayımı sonucu $35^{\circ} \mathrm{C}$ 'da Bakırkoy istasyonunda $9250 \mathrm{KOB} / \mathrm{m}^{3}$ olarak saptanmıştır.

Anahtar Kelimeler: Mikrobiyal fauna, dış ortam havası, sedimentasyon örnekleme, mikrobiyal yük

\section{Investigation of Air Quality in Terms of Microbiological Density in Some Regions of the European Side of Istanbul Province}

\begin{abstract}
Microbial fauna of the outdoor ambient air of Istanbul has been determined in European side of Istanbul province at four different districts (Bakirkoy, Fatih, Basaksehir and Esenler) by active and passive sedimentation sampling methods. In addition to meteorological factors, the effect of urbanization and traffic density for the air microbial load is also determined. Outdoor air sampling was performed between April 2014 and November 2015. The sedimentation method was done by standart plate count agar (PCA) opening the cover of the petri dishes containing a prepared medium. Standart plate count agar (PCA) medium was used for the determination of total bacteria while malt extract agar (MEA) was used for fungi. The colonies formed at the end of this period were counted and total number of bacteria and fungi per unit volume and area were determined. Air temperature, relative humidity, wind speed and direction were also recorded. As a result of the active sampling method, the most counted units of bacteria was determined at $350 \mathrm{C}$ in Basaksehir station as $222 \mathrm{CFU}\left(\right.$ Colony forming unit) $/ \mathrm{m}^{3}$, maximum fungal was determined as $286 \mathrm{CFU} / \mathrm{m}^{3}$ at $20^{\circ} \mathrm{C}$ incubation temperature. The maximum amount of bacteria in the passive sampling method results at $35^{\circ} \mathrm{C}$ was in Bakirkoy station as $9250 \mathrm{CFU} / \mathrm{m}^{3}$.
\end{abstract}

Keywords: Microbial fauna, outdoor air, sedimentation sampling, microbial load

\footnotetext{
* Corresponding Author: Yildiz Technical University, Faculty of Civil Engineering, Environmental Engineering Department, Istanbul, Turkey, ORCID: 0000-0003-4782-4683, hurrembayhan@gmail.com
} 


\section{Introducion}

Several studies have been conducted to determine the number and composition of atmospheric microorganisms. The atmosphere is not suitable for microbial growth due to high light intensity, extreme temperature changes, low organic matter concentration and low water content (Kuzyakov and Razavi, 2019). On the other hand, although the atmosphere is not suitable for the growth of microorganisms, it contains organism at a certain rate. Examples of airborne particles include pollens, microorganisms, and insects. Airborne microorganisms include algae, protozoa. yeasts, molds, rust, bacteria and viruses. The microbial load of air varies based on the amount of dust in the air and the human activities in the environment. Air on top of a fertile soil contains more microorganisms when compared to the air on sandy and muddy soil. Also, compared to the air on an area covered with meadows or forests, the air on a barren land, and compared to the sea air, terrestrial air contains more microorganisms. Air moisture also affects the quantitative status of microorganisms. The main factors behind the degree of bacterial contamination of air are the density of human and animal populations, vegetation, the structure of soil and the earth, the humidity and temperature of the atmosphere, the direction and length of the wind flow (Fujiyoshi et al., 2017).

In the upper layers of the air, aerobic bacilli are more abundant and in the lower layers, there are bacteria groups such as Achromobacter, Sarcina and Micrococcus. These organisms can be transported vertically and horizontally over long distances in the atmosphere. Although autotrophic bacteria could reproduce in clouds where particles that contain gases such as $\mathrm{NH}_{3}$ and $\mathrm{CO}_{2}$ and moisture are present, the growth of pathogenic microorganisms is not possible in the atmosphere (Brown et al., 2018). Bioaerosol is the general name for all airborne organic dust of biological origin such as bacteria, fungi, fungal spores, algae, viruses and pollen and their fragments. Exposure to these biological organisms and their microbial metabolites such as endotoxins, mycotoxins and VOC (Volatile Organic Compounds) leads to adverse health effects (Bennett and Inamdar, 2015). It was reported that in homes, where these types of pollutants are present, the frequency of the crises of asthma patients increases and the pollutants are associated with respiratory diseases (Kim et al., 2018).

The present study aimed to determine the number, composition and seasonal distribution of the total bacteria and fungi in domestic and outdoor atmospheres in Istanbul province and to determine whether the total number of bacterial and fungal colonies were associated with various meteorological factors.

\section{Materials and Methods}

\subsection{Determination of the Stations}

The samples were collected by sedimentation method in Fatih, Bakirkoy, Basaksehir, and Esenler districts in Istanbul (Figure 1) and by active sampling in Bakırkoy, Basaksehir, Esenler districts. The samples analyzed in the study were collected via the 4 stations in European side of Istanbul province between April 2014 - October 2015 in the determined regions for 18 months on certain intervals by active sampling. On September 2015, sampling was conducted by sedimentation method ( $1^{\text {st }}$ station: Bakirkoy, $2^{\text {nd }}$ station: Esenler, $3^{\text {rd }}$ station: Basaksehir, $4^{\text {th }}$ station: Fatih). In each station, total bacterial and fungal colony count were determined with 328 media via sedimentation and filtration methods.

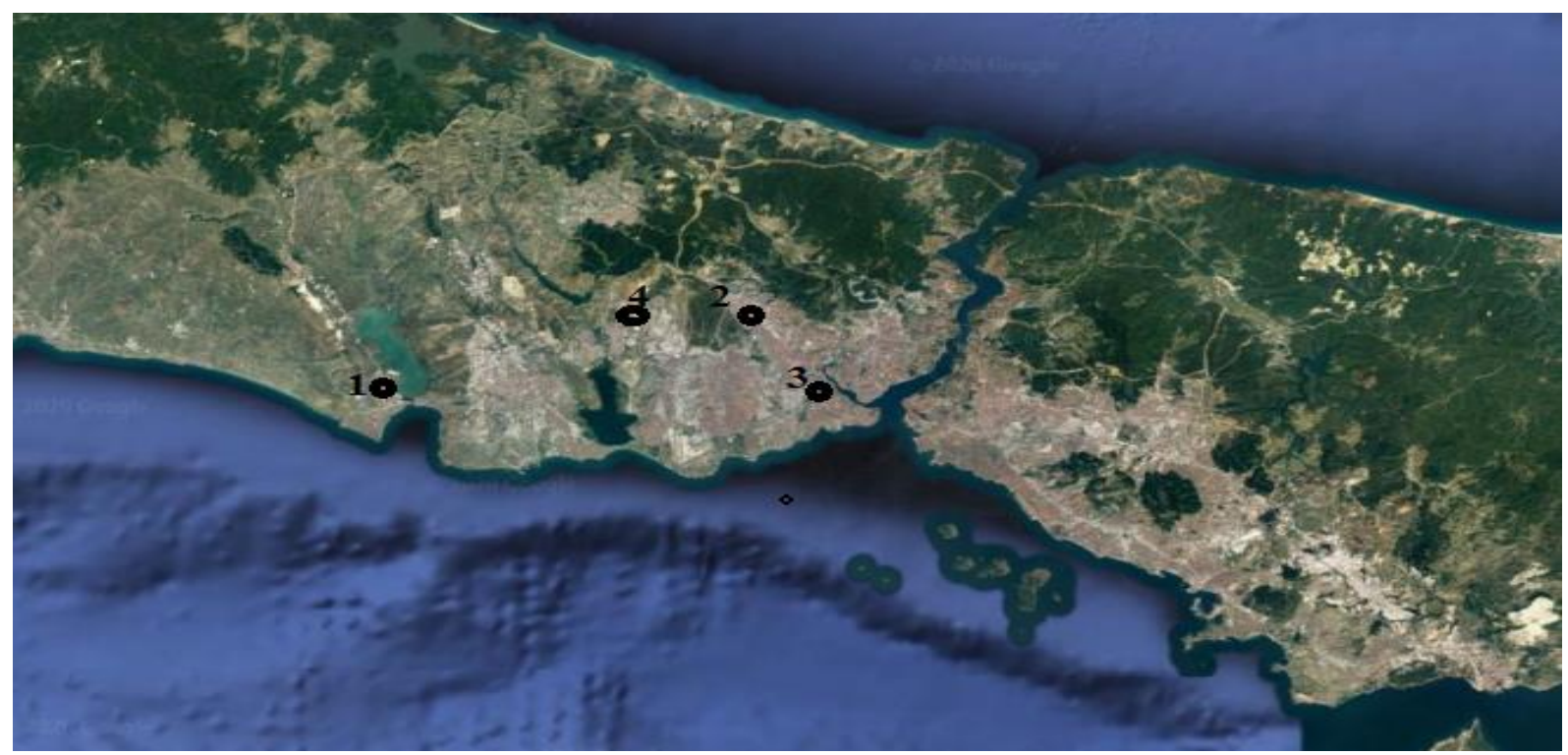

Fig. 1. Sampling stations (1. Station; Bakirkoy, 2. Station; Esenler, 3. Station- Fatih, 4. Station; Basaksehir) 
Bakirkoy station was selected since the district is an urban settlement, urbanization and station transfer center with high traffic load and human population. Basaksehir station was preferred since the district is an organized industrial zone where several activities that cause air pollution are conducted and the district is urban and traffic-intensive center. Fatih station was selected due to high vehicle load and population, while Esenler-Davutpasa station was preferred due to dense plant flora and its windy character. The samples were collected from the outdoor atmosphere with sedimentation and filtration methods. In the present study, the sedimentation method initially developed by Pasteur was used with passive sampling.

\subsection{Active Sampling and Membrane Filtration}

In this method, the outdoor air was collected in the isotonic solution in the gas washing bottle using a vacuum pump. Prior to sampling, the equipment used such as the gas washing bottle, fasteners, isotonic solution, pure water, strainer set, and pipettes were sterilized in an autoclave (Figure 2). Sampling was conducted outdoors at human respiratory level of 1.5 meters. To research the quality of bacteria and fungi present in the air and to determine the suspended solid (SS) content, temperature, humidity, conductivity, samples were collected for 30 minutes in the morning and in the evening at each station at predetermined measurement points. The samples were transported to the laboratory with an ice battery thermos and isolated from the outdoor temperature. Once a specific air volume passed through the filter, the filter that retained all airborne microorganisms was placed on an agar medium. After the membrane filtration and incubation processes, the microbiological load in the ambient air was determined.

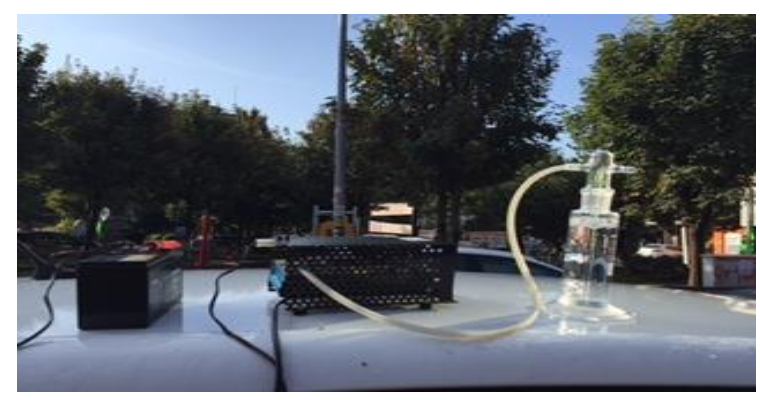

Fig. 2. Active sampling method

\subsection{Sedimentation}

Four petri dishes for total bacteria and for fungi determination were left with the lids off at 1.5 meters high in the sampling area about 30 minutes. Then, the lids were wrapped with stretch film and transferred to the laboratory in an ice battery thermos for incubation. Petri dishes were allowed to incubate for 48 hours at $25^{\circ} \mathrm{C}$ and $35^{\circ} \mathrm{C}$. The colonies that grew at the end of the incubation period were counted.

\subsection{Analysis}

The strainer set was sterilized under a fume hood to prevent contamination. The agar saturated cardboards were placed in the strainer set. The media used are special cardboards saturated with a solution that is precisely formulated for microorganism growth with optimum parameters and could be stored for a long time and could be used by wetting with $3.5 \mathrm{ml}$ sterile water. Standard PCA was used for total bacteria and MEA was used for mold and yeast determination.

The $250 \mathrm{ml}$ samples in the gas washing bottle, 2 bacteria $\left(37^{\circ} \mathrm{C}-20^{\circ} \mathrm{C}\right)$ in $50 \mathrm{ml}, 2$ fungi $\left(37^{\circ} \mathrm{C}-20^{\circ} \mathrm{C}\right)$ were recorded for bacterial and fungi growth in the media in the air samples exposed to incubation for 2 days. The air temperature was also recorded during air quality measurements.

\section{Results}

\subsection{Bacteria and Fungi Colony Counts Determined with Active Sampling Method}

The total organism and fungal colonies obtained by the active sampling method are presented in Tables 1, 2 and 3. After the sampling and incubation conducted in Davutpasa station between April and November, the results of bacteria and fungi colony count are presented in $\mathrm{CFU} / \mathrm{m}^{3}$. In September, it was recorded that bacteria growth was higher as a result of the sampling and analysis conducted during the morning hours. The air temperature was $25^{\circ} \mathrm{C}$ on 02.09 .2014 when the sampling was conducted. The counting result conducted for the medium for the bacteria that underwent $30 \mathrm{~min}$ sampling and incubation at $30^{\circ} \mathrm{C}$ was $7431 \mathrm{CFU} / \mathrm{m}^{3} .833$ $\mathrm{CFU} / \mathrm{m}^{3}$ colonies were counted in the fungi medium. The air temperature was $21^{\circ} \mathrm{C}$ and the incubation temperature was $35^{\circ} \mathrm{C}$.

In April-November period, it was recorded that bacteria and fungi growth was higher as a result of the sampling and analysis conducted in June during the morning hours. The air temperature was $25^{\circ} \mathrm{C}$ on 15.06 .2014 when the sampling was conducted. The counting result conducted for the medium for the bacteria that underwent $30 \mathrm{~min}$ sampling and incubation at $20^{\circ} \mathrm{C}$ was $7500 \mathrm{CFU} / \mathrm{m}^{3}$. The count and analysis conducted on the fungi medium sampling collected on the same date and morning hours and incubated in $20^{\circ} \mathrm{C}$ revealed $1528 \mathrm{CFU} / \mathrm{m}^{3}$ fungi colonies. 
European Journal of Science and Technology

Table 1. Colony counting results at Bakirkoy-Incirli stations via active sampling method.

\begin{tabular}{|c|c|c|c|c|c|c|}
\hline Station & Sampling date & Sampling time & Air temperature & Air pollutants & $\begin{array}{l}\text { Incubation } \\
\text { temperature }\end{array}$ & $\begin{array}{c}\text { Toal colony } \\
\text { number } \\
\left(\mathrm{CFU} / \mathrm{m}^{3}\right)\end{array}$ \\
\hline \multirow{64}{*}{$\begin{array}{l}\text { Bakİrkoy- } \\
\text { Incirli }\end{array}$} & \multirow[t]{4}{*}{25.4 .2014} & \multirow[t]{4}{*}{ 09:00-10:00 } & \multirow[t]{4}{*}{25} & \multirow[t]{2}{*}{ Bacteria } & 20 & 222 \\
\hline & & & & & 35 & 150 \\
\hline & & & & \multirow[t]{2}{*}{ Fungus } & 20 & 45 \\
\hline & & & & & 35 & 27 \\
\hline & \multirow{4}{*}{ 25.4.2014 } & \multirow[t]{4}{*}{ 18:00-19:00 } & \multirow[t]{4}{*}{27} & \multirow[t]{2}{*}{ Bacteria } & 20 & 66 \\
\hline & & & & & 35 & 51 \\
\hline & & & & \multirow[t]{2}{*}{ Fungus } & 20 & 33 \\
\hline & & & & & 35 & 23 \\
\hline & \multirow{4}{*}{ 25.5.2014 } & \multirow[t]{4}{*}{ 09:00-10:00 } & 27 & Bacteria & 20 & 4 \\
\hline & & & & & 35 & 10 \\
\hline & & & & Fungus & 20 & 4 \\
\hline & & & & & 35 & 3 \\
\hline & & 18:00-19:00 & 30 & Bacteria & 20 & 12 \\
\hline & 25.5.2014 & & & & 35 & 5 \\
\hline & & & & Fungus & 20 & 14 \\
\hline & & & & & 35 & 6 \\
\hline & & 09:00-10:00 & 27 & Bacteria & 20 & 6 \\
\hline & 25.6.2014 & & & & 35 & 8 \\
\hline & & & & Fungus & 20 & 6 \\
\hline & & & & & 35 & 4 \\
\hline & & 18:00-19:00 & 30 & Bacteria & 20 & 5 \\
\hline & 25.6.2014 & & & & 35 & 3 \\
\hline & & & & Fungus & 20 & 6 \\
\hline & & & & & 35 & 3 \\
\hline & 25.7.2014 & 09:00-10:00 & 24 & Bacteria & 20 & 4 \\
\hline & & & & & 35 & 4 \\
\hline & & & & Fungus & 20 & 8 \\
\hline & & & & & 35 & 2 \\
\hline & 25.7 .2014 & 18:00-19:00 & 28 & Bacteria & 20 & 2 \\
\hline & & & & & 35 & 2 \\
\hline & & & & Fungus & 20 & 2 \\
\hline & & & & & 35 & 2 \\
\hline & 25.8 .2014 & 09:00-10:00 & 19 & Bacteria & 20 & 21 \\
\hline & & & & & 35 & 14 \\
\hline & & & & Fungus & 20 & 4 \\
\hline & & & & & 35 & 27 \\
\hline & 25.8 .2014 & 18:00-19:00 & 20 & Bacteria & 20 & 47 \\
\hline & & & & & 35 & 27 \\
\hline & & & & Fungus & 20 & 2 \\
\hline & & & & & 35 & 2 \\
\hline & & 09:00-10:00 & 15 & Bacteria & 20 & 5 \\
\hline & & & & & 35 & 2 \\
\hline & 25.9.2014 & & & Fungus & 20 & 7 \\
\hline & & & & & 35 & 3 \\
\hline & & 18:00-19:00 & 15 & Bacteria & 20 & 11 \\
\hline & & & & & 35 & 4 \\
\hline & & & & Fungus & 20 & 286 \\
\hline & & & & & 35 & 2 \\
\hline & 25.10 .2014 & 09:00-10:00 & 16 & Bacteria & 20 & 49 \\
\hline & & & & & 35 & 58 \\
\hline & & & & Fungus & 20 & 19 \\
\hline & & & & & 35 & 6 \\
\hline & 25.10 .2014 & 18:00-19:00 & 18 & Bacteria & 20 & 47 \\
\hline & & & & & 35 & 49 \\
\hline & & & & Fungus & 20 & 8 \\
\hline & & & & & 35 & 6 \\
\hline & 25.11.2014 & 09:00-10:00 & 9 & Bacteria & 20 & 14 \\
\hline & & & & & 35 & 6 \\
\hline & & & & Fungus & 20 & 24 \\
\hline & & & & & 35 & 11 \\
\hline & 25.11 .2014 & 18:00-19:00 & 10 & Bacteria & 20 & 4 \\
\hline & & & & & 35 & 3 \\
\hline & & & & Fungus & 20 & 2 \\
\hline & & & & & 35 & 2 \\
\hline
\end{tabular}


Avrupa Bilim ve Teknoloji Dergisi

Table 2. Colony counting results at Davutpasa-Esenler stations via active sampling method.

\begin{tabular}{|c|c|c|c|c|c|c|}
\hline Station & Sampling date & Sampling time & Air temperature & Air pollutants & $\begin{array}{l}\text { Incubation } \\
\text { temperature }\end{array}$ & $\begin{array}{c}\text { Toal colony } \\
\text { number } \\
\left(\mathrm{CFU} / \mathrm{m}^{3}\right)\end{array}$ \\
\hline \multirow{64}{*}{$\begin{array}{l}\text { Davutpasa- } \\
\text { Esenler }\end{array}$} & \multirow{4}{*}{ 2.4.2014 } & \multirow[t]{4}{*}{ 09:00-10:00 } & \multirow{4}{*}{$15^{0}$} & \multirow[t]{2}{*}{ Bacteria } & 20 & $\mathbf{0}$ \\
\hline & & & & & 35 & 2 \\
\hline & & & & \multirow[t]{2}{*}{ Fungus } & 20 & 23 \\
\hline & & & & & 35 & 19 \\
\hline & \multirow{4}{*}{2.4 .2014} & 18:00-19:00 & \multirow{4}{*}{$17^{0}$} & \multirow[t]{2}{*}{ Bacteria } & 20 & $\mathbf{0}$ \\
\hline & & & & & 35 & $\mathbf{0}$ \\
\hline & & & & \multirow[t]{2}{*}{ Fungus } & 20 & 12 \\
\hline & & & & & 35 & 6 \\
\hline & \multirow{4}{*}{ 2.5.2014 } & 09:00-10:00 & \multirow{4}{*}{$16^{0}$} & \multirow[t]{2}{*}{ Bacteria } & 20 & $\mathbf{0}$ \\
\hline & & & & & 35 & 0 \\
\hline & & & & Fungus & 20 & $\mathbf{0}$ \\
\hline & & & & & 35 & $\mathbf{0}$ \\
\hline & & 18:00-19:00 & & Bacteria & 20 & 10 \\
\hline & 252014 & & $10^{0}$ & & 35 & 14 \\
\hline & 2.5 .2014 & & 19 & Fungus & 20 & 6 \\
\hline & & & & & 35 & 2 \\
\hline & & 09:00-10:00 & & Bacteria & 20 & 103 \\
\hline & 262014 & & 23 & & 35 & 72 \\
\hline & 2.0 .2014 & & 23 & Fungus & 20 & 6 \\
\hline & & & & & 35 & 4 \\
\hline & & 18:00-19:00 & & Bacteria & 20 & 119 \\
\hline & 262014 & & 24 & & 35 & 126 \\
\hline & 2.0 .2014 & & 24 & Fungus & 20 & 10 \\
\hline & & & & & 35 & 6 \\
\hline & & 09:00-10:00 & & Bacteria & 20 & 25 \\
\hline & & & & & 35 & 33 \\
\hline & 2.7 .2014 & & 25 & Fungus & 20 & 2 \\
\hline & & & & & 35 & 2 \\
\hline & 2.7 .2014 & 18:00-19:00 & 29 & Bacteria & 20 & 3 \\
\hline & & & & & 35 & 1 \\
\hline & & & & Fungus & 20 & 2 \\
\hline & & & & & 35 & 2 \\
\hline & 2.8 .2014 & 09:00-10:00 & 27 & Bacteria & 20 & 5 \\
\hline & & & & & 35 & 2 \\
\hline & & & & Fungus & 20 & 2 \\
\hline & & & & & 35 & 2 \\
\hline & 2.8.2014 & 18:00-19:00 & 29 & Bacteria & 20 & 11 \\
\hline & & & & & 35 & 3 \\
\hline & & & & Fungus & 20 & 18 \\
\hline & & & & & 35 & 6 \\
\hline & 2.9.2014 & 09:00-10:00 & 25 & Bacteria & 20 & 177 \\
\hline & & & & & 35 & 220 \\
\hline & & & & Fungus & 20 & 21 \\
\hline & & & & & 35 & 8 \\
\hline & 2.9.2014 & 18:00-19:00 & 29 & Bacteria & 20 & 2 \\
\hline & & & & & 35 & 8 \\
\hline & & & & Fungus & 20 & 4 \\
\hline & & & & & 35 & 4 \\
\hline & 2.10 .2014 & 09:00-10:00 & 21 & Bacteria & 20 & 51 \\
\hline & & & & & 35 & 119 \\
\hline & & & & Fungus & 20 & 6 \\
\hline & & & & & 35 & 25 \\
\hline & 2.10 .2014 & 18:00-19:00 & 23 & Bacteria & 20 & 60 \\
\hline & & & & & 35 & 126 \\
\hline & & & & Fungus & 20 & 12 \\
\hline & & & & & 35 & 5 \\
\hline & 2.11 .2014 & 09:00-10:00 & 14 & Bacteria & 20 & 3 \\
\hline & & & & & 35 & 4 \\
\hline & & & & Fungus & 20 & 5 \\
\hline & & & & & 35 & 6 \\
\hline & 2.11 .2014 & 18:00-19:00 & 17 & Bacteria & 20 & 7 \\
\hline & & & & & 35 & 8 \\
\hline & & & & Fungus & 20 & 9 \\
\hline & & & & & 35 & 10 \\
\hline
\end{tabular}


European Journal of Science and Technology

Table 3. Colony counting results at Ikitelli-Basaksehir stations via active sampling method.

\begin{tabular}{|c|c|c|c|c|c|c|}
\hline Station & Sampling date & Sampling time & Air temperature & Air pollutants & $\begin{array}{c}\text { Incubation } \\
\text { temperature }\end{array}$ & $\begin{array}{c}\text { Toal colony } \\
\text { number } \\
\left(\mathrm{CFU} / \mathrm{m}^{3}\right) \\
\end{array}$ \\
\hline \multirow{60}{*}{$\begin{array}{c}\text { Ikitelli- } \\
\text { Basaksehir }\end{array}$} & \multirow[t]{4}{*}{15.4 .2015} & \multirow[t]{4}{*}{ 09:00-10:00 } & \multirow[t]{4}{*}{12} & \multirow[t]{2}{*}{ Bacteria } & 20 & 6 \\
\hline & & & & & 35 & 4 \\
\hline & & & & \multirow[t]{2}{*}{ Fungus } & 20 & 4 \\
\hline & & & & & 35 & 4 \\
\hline & \multirow[t]{4}{*}{15.4 .2015} & \multirow[t]{4}{*}{ 18:00-19:00 } & \multirow[t]{4}{*}{13} & \multirow[t]{2}{*}{ Bacteria } & 20 & 10 \\
\hline & & & & & 35 & 16 \\
\hline & & & & \multirow[t]{2}{*}{ Fungus } & 20 & 6 \\
\hline & & & & & 35 & 10 \\
\hline & \multirow[t]{4}{*}{ 15.5.2015 } & \multirow[t]{4}{*}{ 09:00-10:00 } & \multirow[t]{4}{*}{19} & \multirow[t]{2}{*}{ Bacteria } & 20 & 37 \\
\hline & & & & & 35 & 41 \\
\hline & & & & Fungus & 20 & 8 \\
\hline & & & & & 35 & 4 \\
\hline & 15.6 .2015 & 09:00-10:00 & 25 & Bacteria & 20 & 222 \\
\hline & & & & & 35 & 150 \\
\hline & & & & Fungus & 20 & 45 \\
\hline & & & & & 35 & 27 \\
\hline & 15.6 .2015 & 18:00-19:00 & 27 & Bacteria & 20 & 66 \\
\hline & & & & & 35 & 51 \\
\hline & & & & Fungus & 20 & 33 \\
\hline & & & & & 35 & 23 \\
\hline & 15.7 .2015 & 09:00-10:00 & 27 & Bacteria & 20 & 4 \\
\hline & & & & & 35 & 10 \\
\hline & & & & Fungus & 20 & 4 \\
\hline & & & & & 35 & 2 \\
\hline & 15.7 .2015 & 18:00-19:00 & 30 & Bacteria & 20 & 19 \\
\hline & & & & & 35 & 7 \\
\hline & & & & Fungus & 20 & 24 \\
\hline & & & & & 35 & 9 \\
\hline & 15.8 .2015 & 09:00-10:00 & 27 & Bacteria & 20 & 6 \\
\hline & & & & & 35 & 8 \\
\hline & & & & Fungus & 20 & 6 \\
\hline & & & & & 35 & 2 \\
\hline & 15.8.2015 & 18:00-19:00 & 30 & Bacteria & 20 & 5 \\
\hline & & & & & 35 & 2 \\
\hline & & & & Fungus & 20 & 11 \\
\hline & & & & & 35 & 3 \\
\hline & 15.9 .2015 & 09:00-10:00 & 24 & Bacteria & 20 & 4 \\
\hline & & & & & 35 & 4 \\
\hline & & & & Fungus & 20 & 8 \\
\hline & & & & & 35 & 2 \\
\hline & 15.9 .2015 & 18:00-19:00 & 28 & Bacteria & 20 & 2 \\
\hline & & & & & 35 & 2 \\
\hline & & & & Fungus & 20 & 2 \\
\hline & & & & & 35 & 2 \\
\hline & 15.10 .2015 & 09:00-10:00 & 19 & Bacteria & 20 & 21 \\
\hline & & & & & 35 & 14 \\
\hline & & & & Fungus & 20 & 4 \\
\hline & & & & & 35 & 27 \\
\hline & 15.10 .2015 & 18:00-19:00 & 20 & Bacteria & 20 & 47 \\
\hline & & & & & 35 & 27 \\
\hline & & & & Fungus & 20 & 2 \\
\hline & & & & & 35 & 2 \\
\hline & 15.11.2015 & 09:00-10:00 & 15 & Bacteria & 20 & 16 \\
\hline & & & & & 35 & 7 \\
\hline & & & & Fungus & 20 & 2 \\
\hline & & & & & 35 & 2 \\
\hline & 15.11.2015 & 18:00-19:00 & 15 & Bacteria & 20 & 9 \\
\hline & & & & & 35 & 2 \\
\hline & & & & Fungus & 20 & 286 \\
\hline & & & & & 35 & 2 \\
\hline
\end{tabular}

\subsection{Bacteria and Fungi Colony Counts Determined with Sedimentation Method}

The total bacteria and fungi colony counts obtained with sedimentation method in Basaksehir- Ikitelli, Davutpasa-Esenler, FatihAksaray, Bakirkoy-Incirli stations are presented in Tables 4, 5, 6 and 7. 
Table 4. Colony counting results at Basaksehir-Ikitelli stations via sedimentation method

\begin{tabular}{|c|c|c|c|c|c|c|}
\hline Station & Sampling date & $\begin{array}{l}\text { Sampling } \\
\text { time }\end{array}$ & Air temperature & Air pollutants & $\begin{array}{c}\text { Incubation } \\
\text { temperature }\end{array}$ & $\begin{array}{c}\text { Toal colony } \\
\text { number } \\
\left(\mathrm{CFU} / \mathrm{m}^{3}\right)\end{array}$ \\
\hline \multirow{24}{*}{$\begin{array}{l}\text { Basaksehir-İkitelli } \\
\text { (sedimentasyon) }\end{array}$} & \multirow[t]{4}{*}{13.9 .2015} & \multirow[t]{4}{*}{ 15:00-16:00 } & \multirow[t]{4}{*}{25} & \multirow[t]{2}{*}{ Bacteria } & 20 & 2563 \\
\hline & & & & & 35 & 1938 \\
\hline & & & & \multirow[t]{2}{*}{ Fungus } & 20 & 250 \\
\hline & & & & & 35 & 250 \\
\hline & \multirow[t]{4}{*}{16.9 .2015} & \multirow[t]{4}{*}{ 16:00-17:00 } & \multirow[t]{4}{*}{25} & \multirow[t]{2}{*}{ Bacteria } & 20 & 313 \\
\hline & & & & & 35 & 1063 \\
\hline & & & & \multirow[t]{2}{*}{ Fungus } & 20 & 324 \\
\hline & & & & & 35 & 63 \\
\hline & \multirow[t]{4}{*}{ 17.9.2015 } & \multirow[t]{4}{*}{ 15:00-16:00 } & \multirow[t]{4}{*}{27} & \multirow[t]{2}{*}{ Bacteria } & 20 & 233 \\
\hline & & & & & 35 & 1063 \\
\hline & & & & \multirow[t]{2}{*}{ Fungus } & 20 & 411 \\
\hline & & & & & 35 & 67 \\
\hline & \multirow[t]{4}{*}{ 21.9.2015 } & \multirow[t]{4}{*}{ 14:00-15:00 } & \multirow[t]{4}{*}{26} & \multirow[t]{2}{*}{ Bacteria } & 20 & 125 \\
\hline & & & & & 35 & 250 \\
\hline & & & & \multirow[t]{2}{*}{ Fungus } & 20 & $\mathbf{7 5 0}$ \\
\hline & & & & & 35 & 156 \\
\hline & \multirow[t]{4}{*}{ 23.9.2015 } & \multirow[t]{4}{*}{ 12:00-13:00 } & \multirow[t]{4}{*}{23} & \multirow[t]{2}{*}{ Bacteria } & 20 & 34 \\
\hline & & & & & 35 & 11 \\
\hline & & & & \multirow[t]{2}{*}{ Fungus } & 20 & 438 \\
\hline & & & & & 35 & 313 \\
\hline & \multirow[t]{4}{*}{30.9 .2015} & \multirow[t]{4}{*}{ 17:00-18:00 } & \multirow[t]{4}{*}{16} & \multirow[t]{2}{*}{ Bacteria } & 20 & 250 \\
\hline & & & & & 35 & 188 \\
\hline & & & & \multirow[t]{2}{*}{ Fungus } & 20 & 313 \\
\hline & & & & & 35 & 63 \\
\hline
\end{tabular}

The sampling conducted with sedimentation method was carried out in September at all stations. The highest value was recorded for the samples collected before 23:00 in Bakirkoy station on 23.09.215. For the fungi, the air temperature was recorded as $25^{0} \mathrm{C}$ before noon on 21.09.2015 for the samples collected in Esenler station. The highest value obtained in total organism colony count was $9250 \mathrm{CFU} / \mathrm{m}^{3}$ and the highest value obtained in total fungus colony count was $875 \mathrm{CFU} / \mathrm{m}^{3}$.

Table 5. Colony counting results at Davutpasa-Esenler stations via sedimentation method

\begin{tabular}{|c|c|c|c|c|c|c|}
\hline Station & Sampling date & $\begin{array}{l}\text { Sampling } \\
\text { time }\end{array}$ & Air temperature & Air pollutants & $\begin{array}{l}\text { Incubation } \\
\text { temperature }\end{array}$ & $\begin{array}{c}\text { Toal colony } \\
\text { number } \\
\left(\mathrm{CFU} / \mathrm{m}^{3}\right)\end{array}$ \\
\hline \multirow{28}{*}{$\begin{array}{c}\text { Davutpasa- } \\
\text { Esenler(sedimentasyon) }\end{array}$} & \multirow[t]{4}{*}{ 7.9.2015 } & \multirow[t]{4}{*}{ 15:00-16:00 } & \multirow[t]{4}{*}{25} & \multirow[t]{2}{*}{ Bacteria } & 20 & 125 \\
\hline & & & & & 35 & 94 \\
\hline & & & & \multirow[t]{2}{*}{ Fungus } & 20 & 188 \\
\hline & & & & & 35 & 438 \\
\hline & \multirow[t]{4}{*}{ 17.9.2015 } & \multirow[t]{4}{*}{$16: 00-17: 00$} & \multirow[t]{4}{*}{26} & \multirow[t]{2}{*}{ Bacteria } & 20 & 220 \\
\hline & & & & & 35 & 42 \\
\hline & & & & \multirow[t]{2}{*}{ Fungus } & 20 & 34 \\
\hline & & & & & 35 & 8 \\
\hline & \multirow[t]{4}{*}{21.9 .2015} & \multirow[t]{4}{*}{ 11:00-12:00 } & \multirow[t]{4}{*}{25} & \multirow[t]{2}{*}{ Bacteria } & 20 & 125 \\
\hline & & & & & 35 & 94 \\
\hline & & & & \multirow[t]{2}{*}{ Fungus } & 20 & 188 \\
\hline & & & & & 35 & 438 \\
\hline & \multirow[t]{4}{*}{29.9 .2015} & \multirow{4}{*}{ 11:00-12:00 } & \multirow[t]{4}{*}{21} & \multirow[t]{2}{*}{ Bacteria } & 20 & 3450 \\
\hline & & & & & 35 & 1125 \\
\hline & & & & \multirow[t]{2}{*}{ Fungus } & 20 & 540 \\
\hline & & & & & 35 & 250 \\
\hline & \multirow[t]{4}{*}{ 21.9.2015 } & \multirow[t]{4}{*}{ 13:00-14:00 } & \multirow[t]{4}{*}{26} & \multirow[t]{2}{*}{ Bacteria } & 20 & 36 \\
\hline & & & & & 35 & 250 \\
\hline & & & & \multirow[t]{2}{*}{ Fungus } & 20 & 438 \\
\hline & & & & & 35 & 625 \\
\hline & \multirow[t]{4}{*}{ 23.9.2015 } & \multirow[t]{4}{*}{ 11:00-12:00 } & \multirow[t]{4}{*}{25} & \multirow[t]{2}{*}{ Bacteria } & 20 & 3000 \\
\hline & & & & & 35 & 9250 \\
\hline & & & & \multirow[t]{2}{*}{ Fungus } & 20 & 750 \\
\hline & & & & & 35 & 250 \\
\hline & 30.9.2015 & 19:00-20:00 & 15 & Bacteria & 20 & 250 \\
\hline & & & & & 35 & 3000 \\
\hline & & & & Fungus & 20 & 438 \\
\hline & & & & & 35 & 125 \\
\hline
\end{tabular}


Table 6. Colony counting results at Fatih-Aksaray stations via sedimentation method.

\begin{tabular}{|c|c|c|c|c|c|c|}
\hline Station & Sampling date & Sampling time & Air temperature & $\begin{array}{c}\text { Air pollutants } \\
\text { Incubation }\end{array}$ & $\begin{array}{c}\text { Toal colony } \\
\text { number } \\
\text { temperature } \\
\text { (CFU/m }\end{array}$ \\
\hline \multirow{5}{*}{ Fatih-Aksaray.) }
\end{tabular}

Table 7. Colony counting results at Bakirkoy-Incirli stations via sedimentation method.

\begin{tabular}{|c|c|c|c|c|c|c|}
\hline Station & Sampling date & Sampling time & $\begin{array}{c}\text { Air } \\
\text { Temperature }\end{array}$ & Air Polltants & $\begin{array}{l}\text { Incubation } \\
\text { temperature }\end{array}$ & $\begin{array}{c}\text { Toal colony } \\
\text { number } \\
\text { (CFU/m } \mathbf{m}^{3} \text { min.) }\end{array}$ \\
\hline \multirow{12}{*}{$\begin{array}{l}\text { Bakirkoy- } \\
\text { Incirli }\end{array}$} & \multirow{4}{*}{21.9 .2015} & \multirow[t]{4}{*}{ 13:00-14:00 } & \multirow[t]{4}{*}{26} & \multirow[t]{2}{*}{ Bacteria } & $20^{0}$ & 63 \\
\hline & & & & & $35^{0}$ & 250 \\
\hline & & & & \multirow[t]{2}{*}{ Fungus } & $20^{0}$ & 438 \\
\hline & & & & & $35^{0}$ & 625 \\
\hline & \multirow[t]{4}{*}{ 23.9.2015 } & \multirow[t]{4}{*}{ 11:00-12.00 } & \multirow[t]{4}{*}{25} & \multirow[t]{2}{*}{ Bacteria } & $20^{0}$ & 3000 \\
\hline & & & & & $35^{0}$ & 9250 \\
\hline & & & & \multirow[t]{2}{*}{ Fungus } & $20^{0}$ & 750 \\
\hline & & & & & $35^{0}$ & 250 \\
\hline & \multirow[t]{4}{*}{ 30.9.2015 } & \multirow[t]{4}{*}{ 19:00-20:00 } & \multirow[t]{4}{*}{15} & \multirow[t]{2}{*}{ Bacteria } & $20^{0}$ & 250 \\
\hline & & & & & $35^{0}$ & 3000 \\
\hline & & & & \multirow[t]{2}{*}{ Fungus } & $20^{0}$ & 438 \\
\hline & & & & & $35^{0}$ & 125 \\
\hline
\end{tabular}

\section{Discussion}

In this study, there is no test for characterization of bacteria/fungus to identify the specific types but ony the approximately number of microorganisms counted for four stations. Studies on determination of bacteria and fungi in indoor/outdoor environments increased significantly during recent years. There are several studies that investigated the presence of bacteria and/or fungi in different types of indoor air, especially in Istanbul, Edirne (Sarica et al., 2002; Asan et al., 2004; Aydogdu et al., 2005; Okten and Asan, 2012) Eskisehir (Asan et al., 2004; Ceylan et al., 2006),Adana (Inal et al., 2007), Manisa (Kalyoncu and Ekmekci, 2008), Isparta (Asan et al., 2004; Unlu et al. (2003), Afyon (Cetinkaya et al., 2005), Denizli (Ovez et al., 2012) and Ankara (Mentese et al., 2012) provinces in Turkey.

In a study conducted at McGill University, it was determined that the correlation between air density and microbial concentration was similar in Montreal and London. Samples were collected from the oceans and islands up to 2700-3000 meter elevations. The analysis of these samples demonstrated that bacteria and fungi could live up to an elevation of 3000 meters. Micrococcus, Sarcina, Gram (-) and Gram (+) bacilli were determined among the aerobic bacterial spore forms. They examined the microbiological flora in the bedroom, living room, kitchen and bathroom of a newly built apartment and in the garden of the same apartment building. For this purpose, both air and surface samples were collected. The air samples were collected at 400 locations in the garden and in each room in the apartment at 2 locations with the petri dishes prepared with Tryptic Soy Agar (TSA) and left open for 10 minutes (Pelczar and Reid, 1965).

In one of the other study, the mycoflora in indoor and outdoor air in the six different districts of the city of Istanbul were investigated at two monthly intervals from November 15, 2001 to September 15, 2002. The aim of that study was to find out the densities of airborne fungal spores that can cause an allergic response on inhalation and/or asthma. The most widespread fungal spores were identified as those of the genus Cladosporium and Penicillium (Colakoglu, 2004). Colakoglu (1996), took outdoor air samples from the Marmara University campus area and a crowded street over a year long period using a volumetric spore trap.

Colakoglu (2004) studied indoor and outdoor mycoflora in the different districts of the city of Istanbul. According to the results, a total of 504 samples were observed by using the Petri Plate Gravitational Method; fungi were isolated from these samples and 2198 colonies were counted. Penicillium was found in greatest abundance followed by Aspergillus, Cladosporium, Alternaria, Rhizopus and Fusarium and Cladosporium and Alternaria. At the end of the study, it was conducted that, there was a high density of the fungi Penicillium and Aspergillus in the indoor air while in the outdoor air, Cladosporium and Alternaria population were high. It is understood that there were differences in the distribution of other fungal species between the indoor and outdoor air samples (Colakoglu, 2004). A similar was study about gravimetric sampling was carried out in Belgrad Forest by Colakoglu, (2003). According to the study, outside the city center, over the course of a year, finding that Aspergillus and Penicillium were the most populated types of fungi. 
The average concentration of bacteria in indoor air was $73 \mathrm{CFU} / \mathrm{m}^{3}$ in the bathroom, $116 \mathrm{CFU} / \mathrm{m}^{3}$ in the bedroom, 140 $\mathrm{CFU} / \mathrm{m}^{3}$ in the living room and $144 \mathrm{CFU} / \mathrm{m}^{3}$ in the kitchen. The mean bacteria count in the house was similar to the outdoor mean bacteria count. There were statistical differences between outdoor bacteria concentrations in the studies conducted on the $1^{\text {st }}$ and $2^{\text {nd }}$ year. Gram (-) bacilli were found on moist surfaces such as kitchen faucets and wash basins, Gram (+) cocci were found on kitchen faucet, bathtubs and faucets. Gram $(+)$ bacilli were found to be higher in both moist and dry surfaces (Marcher and Flores, 1991). The increase in air pollution in Turkey and abroad in recent years and resulting increase in respiratory system diseases especially in humans led to an increasing number of studies on the topic. Khan et al. (1999) conducted a year-long study to investigate the aerial distribution of Aspergillus and other molds in domestic and outdoors air environment. They utilized Andersen sampler and RoseBengal Agar. They compared the fungal species found in indoor and outdoor atmosphere. They investigated whether airborne particles pass through filters based on their density and size. Removal of bacterial and fungal contaminants from the air was succeeded with a rate of $98.7 \%$ in bacteria and up to $67 \%$ in fungi. They reported that the Penicillium species were dominant after removal and $4 \mu \mathrm{m}$ particles passed through the filters. Sapan et al. (1991) investigated the indoors fungal flora in the province of Bursa. It was found that reproduction was observed in 1014 out of the 1188 media and Penicillium, Cladosporium, Rhizopus ehrenberger, Aspergillus, Absidia tiegh and Mucor mich. Sapan et al. (1993) conducted a study to investigate the fungal flora in the homes of children with bronchial asthma in Bursa. In this study, samples collected at 6 stations for 12 months using Gravity-Based Petri plate method to determine the airborne fungi in Edirne province and determined that the dominant species were Alternaria, Penicillium, and Cladosporium. They also examined the correlation between the number of fungal spores, air pollutants and meteorological factors using statistical analyzes, and found that the correlations between the number of spores and $\mathrm{SO}_{2}$ concentration in the air and temperature were statistically significant. Although this finding contradicted with the findings reported by Bicakci et al. (2001), it was considered that the increase in the temperature, precipitation and humidity provided the optimum conditions for the growth of fungi. The density of microfungi is also high in humid environments.

\section{Conclusions}

In the study, it was observed that in November, although the humidity rate and rainfall were high, the spore count decreased significantly as a result of the decrease in temperatures. The findings of the present study in these months were similar to those reported by other studies. In April and May, it was observed that the spores started to decrease in the atmosphere. It is suggested that this decrease was due to the increasing temperatures and related decrease in relative humidity despite the increase in precipitation in these months. The optimum conditions may vary based on the geographic conditions and climate. In May, temperatures, precipitation and humidity reached suitable levels for these spores to grow and mean monthly temperature was observed as $14.2^{\circ} \mathrm{C}$. It was reported that during the days after the rain, the increase in temperatures and wind speed increase the spore count. It is understood that sampling areas of Istanbul City has a problem about fungal and bacterial load for human health. It is necessary to control microorganism populations in for protect people health from allergic diseases. Those with allergies to fungal spores are recommended not to leave home unless they used to, because organic plant waste concentrations are high and so fungal development is much higher. Scientists are advised to study the range of seasons described above when considering their treatments.

\section{Acknowledge}

This study was produced from master thesis and supported by Yıldız Technical University Scientific Research Projects Coordinator (Project No: 2012-05-02-YL-02).

\section{References}

Asan, A., Ilhan, S., Sen, B., Erkara, I.P., Filik, C., Cabuk, A., Demirel, R., Ture, M., Okten, S.S. \& Tokur, S. (2004). Airborne fungi and actinomycetes concentrations in the air of Eskisehir city (Turkey). Indoor Bullt. Environ., 13, 63-74.

Aydogdu, H., Asan, A., Oktun, M.T. \& Ture, M. (2005). Monitoring of fungi and bacteria in the indoor of primary school in Edirne city, Turkey.; Indoor. Built. Environ., 14, 411-425.

Bennett, J., Inamdar, J. (2015). Are Some Fungal Volatile Organic Compounds (VOCs) Mycotoxins? Toxins (Basel). 7(9), $3785-3804$.

Brown, S.R.B., Forauer, E.C., D'Amico, D.J. (2017). Effect of modified atmosphere packaging on the growth of spoilage microorganisms and Listeria monocytogenes on fresh cheese. J Dairy Sci., 101(9), 7768-7779.

Bicakci, A., Tatlidil, S., Canitez, Y. \& Malyer, H. (2001). Mustafakemalpasa ilcesi (Bursa) atmosferindeki alerjen Alternaria sp. ve Cladosporium sp. sporları, Akciğer Arşivi, 2, 69-72.

Cetinkaya, Z., Fidan, F., Unlu, M., Hasenekoglu, I., Tetik, L. \& Demirel, R. (2005). Assessment of indoor air fungi in WesternAnatolia, Turkey. Asian Pac. J. Allergy. Immunol, 23, 87-92.

Ceylan, E., Ozkutuk, A., Ergor, G., Yucesoy, M., Itil, O., Caymaz, S. \& Cimrin, A. (2006). Fungi and indoor conditions in asthma patients. Journal of Asthma, 43, 789-794.

Colakoglu, G. (1996). Mould Counts in the Atmosphere at the Europe Quarter of İstanbul, Turkey. J Basic Microbiolol., 36(6), 389392.

Colakoglu, G. (2003). Airborne fungal spores at the Belgrad forest near the city of Istanbul (Turkey) in the year 2001 and their relation to allergic diseases. J Basic Microbiolol., 43(5), 376-84. 
Colakoglu, G. (2004). Indoor and outdoor mycoflora in the different districts of the city of Istanbul (Turkey). Indoor. Built Environ. 13(2), 91-100.

Fujiyoshi, S., Tanaka, D., Maruyama, F. (2017). Transmission of Airborne Bacteria across Built Environments and Its Measurement Standards: A Review. Front Microbiol., 8, 2336.

Inal, A., Karakoc, G.B. \& Altintas, D.U. (2007). Effect of indoor mold concentrations on daily symptom severity of children with asthma and/or rhinitis monosensitized to molds. J. Asthma, 44, 543-546.

Kalyoncu, F. \& Ekmekci, S. (2008). Culturable airborne fungi in outdoor environments in Manisa, Turkey. Fresen. Environ. Bull., 17, 844-848.

Khan, Z.U., Khan, M.A., Chandy, R.\& Sharma, P.N. (1999). Aspergillus and other moulds in the air of Kuwait. Mycopathologia, 146(1), 25-32.

Kim, D., Chen, Z., Zhou, L.F., Huang, S.X. (2018). Air pollutants and early origins of respiratory diseases. Chronic Dis Transl Med. $4(2), 75-94$.

Kuzyakov, Y., Razavi, B.S. (2019). Rhizosphere size and shape: Temporal dynamics and spatial stationarity. Soil Biol Biochem. 135, 343-360.

Marcher, J.M. \& Flores, M.A. (1991). Two-Year Study of Miciobiological Indoor Air Quality in a New Apartment, Archives of Environ. Health.. 46(1), 25-29.

Mentese, S., Rad, A., Arisoy, M. \& Gullu, G. (2012). Seasonal and spatial variations of bioaerosols in indoor urban environments, Ankara, Turkey. Indoor Built. Environ., 21, 797-810.

Okten, S. \& Asan, A. (2012). Airborne fungi and bacteria in indoor and outdoor environment of the pediatric unit of Edirne government hospital. Environ. Monit. Assess, 184, 1739-1751.

Ovez, H., Ergin, C. \& Kaleli, I. (2012). Investigation of mold fungi in air samples of elementary schools and evaluation of allergenspecific Ige levels in students' sera. Mikrobiyoloji Bulteni, 46, 266-275.

Pelczar, M.J. \& Reid, R.D. (1965). Microbiology (2nd ed). Kogakuscha Company Ltd, Tokyo.

Sapan, N., Gedıkoglu, S. \& Anturan, N. (1993). Bursa'daki Bronşial Astmalı Çoçukların Evlerindeki Mantar Florasının Belirlenmesi. Akdeniz Üniversitesi Tip Fakültesi Dergisi, 10, 9-12.

Sapan, N., Gedıkoglu, S. \& Tunali, S. (1991). Bursa ili eviçi mantar florası. Türk Mikrobiyol. Cem. Derg., 21, 73-78.

Sarica, S., Asan, A., Oktun, M.T. \& Ture, M. (2002). Monitoring indoor airborne fungi and bacteria in the different areas of Trakya University Hospital, Edirne, Turkey. Indoor Built. Environ., 11, 285-292.

Unlu, M., Ergin, C. \& Cirit, M. (2003). Molds in the homes of asthmatic patients in Isparta, Turkey. . Asian Pac. J. Allergy. Immunol, $21,21-24$. 